\title{
Responsabilidad Social en el fútbol profesional español ¿Una relación para el desarrollo de un nuevo rol ciudadano?
}

\section{Corporate Social Responsibility in Spanish professional soccer: a relationship for the development of a new citizenship?}

\author{
Dra. Isabel Ruiz-Mora es profesora e investigadora de la Universidad de Málaga (España) (isabelruiz@uma.es) \\ (https://orcid.org/0000-0003-0723-9850).
}

Dr. Daniel Guerrero-Navarro es profesor e investigador de la Universidad de Málaga (España) (dguerrero@uma.es) (https://orcid.org/0000-0001-6563-3851).

\begin{abstract}
Resumen
El presente trabajo se enmarca en la relación que se establece entre la Responsabilidad Social de las empresas (RSE) y relaciones públicas en las organizaciones, visto desde el enfoque de la Teoría de la Legitimación (Lindblom, 1994). Se plantea determinar si los clubes profesionales del fútbol español apuestan por la RSE como estrategia para legitimar su rol de ciudadano corporativo en la sociedad. Como objetivos específicos se pretende (1) conocer cuál es la situación de la RSE en los clubes; (2) determinar las políticas de RSE más habituales y, (3) identificar los principales canales de relación y diálogo con los stakeholders. La metodología empleada, con un carácter descriptivo-interpretativo, se focalizará en el estudio de los 42 clubes que componen la Liga de Fútbol Profesional (temporada 2017/2018), de primera y segunda división. Para ello se analizan las memorias anuales, las webs corporativas y las fundaciones de los clubes, por constituirse éstas como instrumentos para implementar políticas de RSE. Los resultados arrojan un escaso compromiso con la RSE, donde se enfatiza la acción social, las acciones destinadas a los socios y el cumplimento con la Ley de Transparencia. La conclusión principal demuestra que los clubes profesionales se limitan a cumplir con la legislación y no tienen un compromiso real con la transparencia y la RSE. El concepto de RSE no es aplicable al sector del fútbol profesional en España.
\end{abstract}

\begin{abstract}
This research explores the relation existing between Corporate Social Responsibility (CSR) and Public Relations (PR) through the legitimization theory (Lindblom, 1994). With this project we aim to determine if soccer clubs are implementing Corporate Social Responsibility (CSR) programs to legitimate their role in society as corporate citizens. The specific objectives are (1) to know how CSR programs are being introduced in Spanish soccer clubs; (2) to determine their main CSR polices and (3) to identify dialogue channels with stakeholders. The methodology is based on the interpretative and descriptive analysis of 42 professional soccer clubs in the Spanish League (2016/2017), including those in the first and second division. In particular, we will analyze annual reports, their corporate websites and finally, the foundations of these clubs as instruments to develop CSR policies. The results show little commitment to CSR, which emphasizes charity, actions aimed at the members and compliance with Transparency Laws. The main conclusion is that professional clubs are limited to comply with the legislation and do not have a real commitment to transparency and CSR. The concept of CSR is not applicable to the professional soccer sector in Spain.
\end{abstract}

\section{Palabras clave I keywords}

RSE, relaciones con los públicos, ciudadanía, deporte, clubes fútbol, España CSR, public relations, citizenship, sport, football clubs, Spain

Cómo citar: Ruiz-Mora, I. \& Guerrero-Navarro, D. (2018). Responsabilidad Social en el fútbol profesional español ¿Una relación para el desarrollo de un nuevo rol ciudadano?. Retos Revista de Ciencias de la Administración y Economía, 8(16), 61-77. https://doi.org/10.17163/ret.n15.2018.05 


\section{Introducción y estado de la cuestión}

El objetivo principal de este trabajo es el de determinar si los clubes profesionales del fútbol español apuestan por la Responsabilidad Social de las empresas (RSE) como estrategia para legitimar su rol de ciudadano corporativo en la sociedad.

La relación que se establece entre futbol y sociedad se podría abordar desde diferentes ópticas, pero es la RSE la que nos ofrecerá un contexto más integrador y en consonancia con la sociedad actual, que permite observar la relación desde el compromiso, la responsabilidad y el entendimiento. La propia UEFA (2017), organismo rector de este deporte en Europa, sostiene en su página web que:

El fútbol, como parte integral de la sociedad global y como deporte más popular del mundo, tiene un papel inestimable para impulsar el desarrollo social y ejercer su influencia. Bajo el lema de RESPECT, la responsabilidad social de la UEFA se esfuerza en promover el desarrollo sostenible en todos los aspectos del fútbol en cooperación con las otras partes interesadas. Esto no es a expensas de los beneficios, sino en cómo se obtienen estos beneficios.

Alineado con ese mensaje, la propia «Liga» española de fútbol enfoca a cada uno de los actores de la sociedad, incidiendo en los ciudadanos, los aficionados, para transmitirles a través de su Guía Práctica para Aficionados, un mensaje relevante de responsabilidad: «es importante que asumas tus responsabilidades y defiendas los valores fundamentales del fútbol, del deporte, y en particular de nuestras competiciones porque tú eres parte imprescindible e insustituible en el fútbol» (La Liga, 2015).

En la búsqueda de referentes, se resalta cómo Olabe (2012) aborda la RSE como factor de reputación en los clubes de fútbol profesionales, un activo asociado a las emociones que genera. Por su parte, el trabajo de López-Martínez y Fernández-Fernández aborda cómo los clubes de fútbol profesionales «se han convertido en verdaderas empresas que, en muchos casos, no se gestionan conforme a los imperativos legales y éticos exigidos para el resto» (2015, p. 39). La gestión de estas empresas bajo unos estándares de transparencia (ética empresarial), prácticas de Buen Gobierno y RSE se plantea como la vía para luchar contra «una gestión poco seria y rigurosa, con deudas intolerables en cualquier otro sector» (López-Martínez, \& Fernández-Fernández, 2015, p. 40).

Sería difícil entender que un sector como el del fútbol profesional, que genera millones de euros, con gran impacto en la sociedad y que representa un reflejo formativo para muchos jóvenes y niños (Silva-Santos et al., 2014), no potencie el compromiso con la transparencia, la rendición de cuentas y el deber con la sociedad, al amparo de la gestión basada en la RSE. No podemos obviar el rol que los propios jugadores tienen en la sociedad y cómo el futbol influye dentro y fuera del campo de juego (Cano-Tenorio, \& Paniagua-Rojano, 2017; Cano-Tenorio, 2017). Como mencionan Castillo, Fernández, \& Castillero (2016, p. 215) «la particularidad del fútbol como espectáculo moderno le hace disponer de millones de seguidores de todo el mundo» y por tanto, este impacto mediático y social no puede eludir su responsabilidad con la sociedad. No es posible, en este sentido, olvidar la masa tan heterogénea que conforma a los denominados «seguidores», donde gran parte de los mismos está integrada por niños, adolescentes y jóvenes que ven en el futbol y más concretamente, en los futbolistas, fuentes de inspiración a los que quieren imitar. 
De hecho, es consciente «La Liga» de esa responsabilidad social y por ello, a través de su Dirección de Integridad y Seguridad, junto al Consejo Superior de Deportes, lidera el compromiso social, editando la Guía de Buenas Prácticas. Normativa y principios para las federaciones, ligas y deportistas españoles (La Liga, 2015). En este contexto, el Mapa de la responsabilidad social del fútbol profesional español, es publicado por la Fundación de Fútbol Profesional (La Liga, 2017), como «una oportunidad real para agregar valor social al mundo del fútbol profesional (...) y superar de manera conjunta las debilidades que hemos detectado». Fruto de este trabajo, plantean un acercamiento a la RSE desde la labor conjunta de clubes y fundaciones y en el desarrollo de los conceptos Fair Play Social y Fair Play Financiero, como medios para alcanzar un equilibrio económico y social. En este ámbito, plantean el modelo de la ISO $26000^{1}$ para el desarrollo de acciones y actividades de RSE. De hecho, se entrevé en la siguiente afirmación, cómo el mundo del fútbol español ha detectado que la RSE representa una oportunidad también para su negocio: «hemos evidenciado que cuanta más disminución-control de la deuda pública de los clubes, más incremento de su acción social y, a más RS mejor impacto sobre los datos económicos» (La Liga, 2017); la cuestión será entonces averiguar si verdaderamente se produce un compromiso con la RSE y pasa a formar parte de las estrategias de gestión de las organizaciones deportivas.

\subsection{RSE y Relaciones Públicas}

Este trabajo se enmarca conceptualmente en la relación que se establece entre RSE y Relaciones Públicas. Según el Instituto Ethos de Empresas y Responsabilidad Social (2017, p.17), la RSE es:

[...] una forma de gestión que se define por la relación ética de la empresa con todos los públicos con los cuales ella se relaciona y por el establecimiento de metas empresariales compatibles con el desarrollo sostenible de la sociedad, preservando recursos ambientales y culturales para las generaciones futuras, respetando la diversidad y promoviendo la reducción de las desigualdades sociales.

En este orden de ideas, se patenta cómo las empresas deben mantener una relación (ética) con sus públicos, basada en el diálogo, teniendo en cuenta sus intereses y demandas; por tanto, un acercamiento desde las Relaciones Públicas es posible.

La gestión de las relaciones con los públicos se postula como una de las principales funciones de las Relaciones Públicas (Harlow, 1975²; Seitel, 2002; Grunig, \& Hunt, 2003) y éstas, se convierten en un elemento necesario para la correcta gestión y desarrollo de la RSE; además de convertirse en un elemento cohesionador de la RSE al propiciar una relación entre la organización con sus stakeholders. Abordar la gestión de la RSE desde las Relaciones Públicas es inevitable, ya que la gestión de las relaciones con los stakeholders está tanto en el corazón de la RSE, como en las funciones de las Relaciones Públicas (Wang, \& Chaudhri, 2009, p. 247).

Para abordar teóricamente este planteamiento, se trabajará bajo el enfoque de la «Teoría de la Legitimación» (Lindblom, 1994), que afirma que las compañías esta-

\footnotetext{
1 https://goo.gl/jqcGU9

2 Citado en Seitel (2002, p.12).
} 
blecen un contrato explícito o implícito con la sociedad (Shocker, \& Sethi, 1973). Estos autores señalan que toda institución social opera en virtud a un contrato social del que depende su supervivencia y desarrollo y que por tanto, justifica su existencia, por lo que «esta situación obliga a la empresa a una búsqueda constante de su legitimidad, adaptándose a los requerimientos sociales y demostrando su utilidad y necesidad de su existencia» (Ángel-Vega, 2009, p.39).

Según O’Donovan (2002, p. 344), «la teoría de la legitimidad se basa en la idea de que para seguir funcionando con éxito, las empresas deben actuar dentro de los límites de lo que la sociedad identifica como comportamiento aceptable», mientras que Lindblom (1994, p. 2) define la legitimidad como:

Una condición o estado que existe cuando el sistema de valores de una entidad es congruente con el sistema de valores del sistema social más amplio de que la entidad forma parte. Cuando una disparidad, real o potencial, existe entre los dos sistemas de valores, hay una amenaza para la legitimidad de la entidad.

Por medio de la búsqueda de la legitimidad, las organizaciones dan respuesta a las expectativas que los stakeholders tienen sobre ellas mismas. Este contrato social se ve representado en el concepto de "ciudadanía corporativa» (Capriotti, 2006), en el que las empresas se implican en la sociedad como un ciudadano más, y donde los valores corporativos se alinean también con los valores de la sociedad.

Este planteamiento desde las Relaciones Públicas se ve reforzado con otros enfoques que trabajan la importancia de la gestión de las relaciones con los públicos para la supervivencia de la organización desde la óptica de la RSE, concretamente en esfuerzos teóricos como la «Teoría de los Stakeholders» de Freeman (1984), la «Teoría de la Pirámide de la RSE» de Carrol (1999) y el «Enfoque Ético de la RSE» de Cortina (1994). La «Teoría de los Stakeholders» (Freeman, 1984), plantea:

La existencia de una amplia gama de participantes interesados en las empresas y también muestra la condición relacional entre empresa y sociedad. No se trata de dos entidades separadas, sino de dos componentes de un mismo telar que, al tejerse o interactuar, generan un capital o una ganancia denominada capital social. Lo realmente valioso es la relación, que requiere confianza mutua, sólo alcanzable a través del diálogo franco y honesto (Castillo, 2009, p. 235).

Este enfoque sugiere que si adoptamos como unidad de análisis las relaciones entre una empresa y los grupos e individuos que pueden afectar o ser afectados por ella (conocidos como stakeholders), tenemos una mejor oportunidad para hacer frente a los problemas/tensiones que puedan surgir entre ellos (Freeman et al., 2010, p. 5).

De acuerdo con Carroll (1999) y la «Teoría de la Pirámide de la RSE», debemos considerar cuatro niveles de RSE. Estos niveles son (Carroll, 1999; Castillo, 2009, p. 225):

- Responsabilidad económica, el principal objetivo es la maximización del beneficio derivado de su actividad económica.

- Responsabilidad legal, la empresa actúa de acuerdo con las leyes y normas que debe cumplir. 
- Responsabilidad ética, la empresa debe satisfacer las expectativas que los stakeholders tienen respecto a su comportamiento social, laboral y medioambiental. Debe actuar de forma correcta y justa, evitando el «daño».

- Responsabilidad filantrópica, la empresa actúa como «buen ciudadano» y se compromete a contribuir a la mejora del bienestar de la sociedad. Este nivel no implica una obligación y este estado se adquiere cuando se han cumplido los tres anteriores.

Por su parte, Carrol (1999) desarrolló una estrategia de la RSE que aunaba por un lado la maximización de los beneficios cumpliendo con la ley (versión funcionalista), con la ética de los negocios y las responsabilidades de las empresas hacia la sociedad (Raufflet et al., 2012, p. 34).

Cortina (1994), como referente del enfoque ético en la RSE, define la ética como un «tipo de saber de los que pretende orientar la acción humana en un sentido racional (...) para actuar de un modo racional» (1994, p.17) que guía la acción para tomar decisiones prudentes y moralmente justas que nos haga felices (Cortina, 1994, p.18). En el ámbito de la organización, la búsqueda de esa felicidad se interpreta como un fin social, entendido como los bienes/servicios que proporciona a la sociedad y que legitima su existencia (Cortina, 1994, p. 22).

La RSE se fundamenta en la afirmación de que las empresas, en la medida en que actúan libre y voluntariamente, están integradas por diferentes personas que viven en una sociedad con una determinada conciencia y una ética. Son, por tanto, responsables (Navarro, 2008, p. 44).

Como hemos visto, la empresa tiene un fin social como ciudadano. Cuando no cumple las premisas que legitiman su presencia en la sociedad, la ética en la organización orienta a la empresa hacia lo que debería ser (Navarro, 2008, p.45). Para García Marzá, «la ética empresarial no es una parte de la RSE (...) la responsabilidad es una parte de la ética empresarial ocupada de las condiciones que subyacen a la confianza depositada en la empresa» (2007, p.186).

\section{Material y métodos}

La hipótesis de este trabajo plantea que los clubes apuestan por acciones de RSE como una vía para legitimar su rol en la sociedad como ciudadano corporativo. Guiados por los objetivos de la presente investigación, se plantea responder a las siguientes cuestiones:

- ¿Cuál es la situación de la RSE en los clubes españoles de fútbol?

- ¿Cuáles son las políticas de RSE más habituales en estos clubes?

- ¿Cuáles son los principales canales de relación y diálogo empleados por estas organizaciones para gestionar las relaciones con sus stakeholders?

La metodología empleada con un carácter descriptivo-interpretativo, se focaliza en el estudio de los 42 clubes que componen la Liga de Fútbol Profesional (temporada 
2017/2018, datos 2016/2017), de primera ${ }^{3}$ (20) y segunda ${ }^{4}$ (22) división. Realizaremos un análisis de contenido y en profundidad de las siguientes áreas:

- Memorias anuales, por ser identificados como elemento para cumplir con el criterio exigido por la Ley de la Transparencia del Fútbol (Ley 19/2013, de 9 de diciembre, de Transparencia).

- Webs corporativas de dichos clubes, por constituir el principal cauce de relación activa con los públicos y propiciar un contexto favorable para el diálogo.

- Fundaciones de los clubes, por constituirse como instrumentos que buscan el interés general (AEF, s.f., p. 3), suponiendo un instrumento para implementar políticas de RSE, desde la evolución de clubes deportivos a sociedades anónimas deportivas (Ley 10/1990, de 15 de octubre, del Deporte y Real Decreto 1251/1999, de 16 de julio, sobre sociedades anónimas deportivas).

Para ello, se ha diseñado una ficha de análisis que permitirá identificar si la RSE forma parte o no de la estrategia de clubes, así como políticas y acciones de RSE y las herramientas de diálogo y participación. Las principales dimensiones de análisis son:

- Gobierno corporativo y transparencia.

- Herramientas de relación con stakeholders.

- Políticas/líneas estratégicas RSE.

- Información sobre fundaciones.

El acceso a la información se realizó a través del contenido disponible en la web corporativa de cada club. La recogida y análisis de los datos se efectuó durante el primer trimestre del año 2018 y se aplicó un pretest a un 9,5\% de los clubes objeto de estudio. Se consideran elementos fundamentes para que un club se incluya en el proceso de análisis cumplir con 2 de los 3 criterios mínimos:

- Que el club cuente con una web corporativa.

- Que en dicha web, aparezca información sobre el cumplimento con la Ley de Transparencia.

- Que en la web se ofrezca información sobre la Fundación del club.

Si un club no ofrece información sobre el cumplimento con la Ley de Transparencia y su Fundación, es excluido por no ofrecer datos de interés para la presente investigación. Igualmente, consideramos la web y su contenido, como una plataforma de ejercicio de la transparencia, empleada como herramienta de comunicación donde se publica la información de interés y utilidad para el usuario, sin necesidad de que éste deba indagar por su cuenta. Por tanto, el análisis en su totalidad se centra en el contenido que los clubes publican en sus webs.

3 Clubs primera división, Liga Santander. https://goo.gl/SqRquh

4 Clubs segunda división, Liga 1|2|3. https://goo.gl/vxV4Tf 


\section{Análisis y resultados}

Todos los clubes de primera división ofrecen en su web oficial información referente al cumplimiento con la Ley de Transparencia, a excepción de Atlético de Madrid, que ofrece la memoria de la temporada anterior; y Getafe, que posee el directorio y enlace, pero que no dirige a ningún documento.

Solo el 60\% de los clubes (12) ofrecen datos económicos acerca de su Fundación en la web oficial del club. Los que no los ofrecen son: Deportivo Alavés, Atlético de Madrid, Deportivo La Coruña, Getafe FC, Girona FC, Club Deportivo Leganés, Real Celta de Vigo y Villarreal CF.

Los clubes de Segunda División: Albacete Balompié, Lorca Fútbol Club y Club de Fútbol Reus Deportiu son excluidos del análisis por no ofrecer en su web información referente al cumplimento con la Ley de Transparencia e información sobre la fundación. En un solo caso (Granada Club de Fútbol SAD), los datos no están actualizados y en otro, (Cultural y Deportiva Leonesa SAD), no aparecen datos para cumplir con la ley de transparencia, pero en sendos casos, se incluye información sobre las fundaciones de ambos clubes.

En siete casos, no se ofrece información sobre la fundación en la web (independientemente de que cuenten con una), pero sí se facilitan datos sobre el cumplimiento con la ley de transparencia. Concretamente nos referimos a Agrupación Deportiva Alcorcón SAD, Córdoba Club de Fútbol SAD, Granada Club de Fútbol SAD, Club Deportivo Lugo SAD, Gimnàstic de Tarragona SAD, Real Oviedo SAD y Club Deportivo Tenerife SAD. Por lo tanto, el análisis de los clubes de segunda se ha centrado en un corpus de 17 clubes $^{5}$.

\subsection{Ley Transparencia}

En el 90\% de los casos de clubes de primera y en el 76,5\% de los casos de clubes de segunda, la memoria es descargable; mientras que en un $10 \%$ y $23,5 \%$, respectivamente, se apuesta por colgar la información en la propia web. En primera división, en la mitad de los clubes hay resúmenes abreviados. No obstante, en ningún caso en segunda nos encontramos con informes abreviados o resúmenes ejecutivos que ayuden en la rápida lectura, identificación de datos clave e interpretación. Igualmente, sorprende la ausencia en el 100\% de los casos de páginas webs accesibles y adaptadas según protocolos de accesibilidad ${ }^{6}$. En cuanto a los datos de contactos, aparecen en un 76,5\% de los casos en segunda y un testimonial 5\% en primera. El índice de contenidos aparece en un $75 \%$ y un $71 \%$, así como en un $50 \%$ y un $53 \%$ el índice está vinculado al contenido de la memoria, facilitando la navegación al usuario, así como la rápida identificación del contenido de interés. Sólo en el 35\% de los casos de primera división y el 12\% de los casos de segunda división se cuenta con una presentación. En el 30\% de los casos de primera y en ningún caso en segunda división nos encontramos con mensaje de bienvenida del presidente del club.

\footnotetext{
5 Los datos de FC Barcelona B y Sevilla AT no se han incluido en el análisis de los clubes de Segunda División por estar presente los primeros equipos de cada club en los datos de Primera División.

6 Web Accessibility Initiative (WAI). https://goo.gl/8nUjyf
} 
En el pleno de los clubes de máxima categoría y en un 94\% de los casos de segunda división, aparece información corporativa de las entidades en la sección. Esto se interpreta debido, principalmente, a que tienen un marcado carácter estático y su contenido no se adapta con mucha definición al referido en las secciones. Entre el contenido corporativo, destacamos:

- $\quad$ Organigrama (100\% y 94\%, respectivamente).

- Historia y/o antecedentes (100\% y $88 \%)$.

- Hitos, logros alcanzados, palmarés (100\% y 35\%).

- $\quad$ Marca, logo, publicidad (20\% y $82 \%)$.

- Patrocinadores del club (70\% y 94\%).

- $\quad H i m n o(35 \%$ y $47 \%)$.

- $\quad$ Estadio (70\% y 82\%).

- Otros: tienda virtual, venta de entradas, museo, mascota, promociones, instalaciones, antiguos presidentes, ciudad deportiva, agenda, jugadores históricos, manual de imagen corporativa, etc.

\subsection{Gobierno corporativo}

En primera división, el total de los clubes presenta la estructura del consejo de gobierno o administración, y en la mitad de los casos, encontramos participación de mujeres, pero no se menciona explícitamente en ningún caso la representación masculina frente a la femenina. En el 30\% se mencionan los cargos que los miembros del consejo ocupan fuera del club, en el 65\% se menciona la remuneración (un 35\% del total menciona explícitamente que no percibe remuneración por su cargo en la sociedad). En el 80\% de los casos, se alude al código ético.

En segunda división, en un $82 \%$ se presenta la estructura del consejo de gobierno/ consejo de administración, sólo en tres casos encontramos una mujer y en un caso, dos mujeres. En consecuencia, en ningún caso se menciona explícitamente la representación masculina frente a la femenina. En el 60\% se mencionan los cargos que los miembros del consejo ocupan fuera del club, así como la remuneración. En el $47 \%$ se alude al código ético.

\subsection{Stakeholders y herramientas de comunicación}

En referencia a los grupos de interés de los clubes analizados en primera división, podemos destacar que los principales que aparecen son:

- Peñas $(100 \%)$.

- Aficionados (100\%).

- Abonados (100\%).

- Fans/seguidores (100\%).

- Accionistas (90\%).

- Patrocinadores (65\%\%).

- Medios de comunicación (35\%). 
- Administración local / gobierno (55\%).

- Comunidad y sociedad en general (40\%).

- Sector educativo (35\%).

- Sector profesional (20\%).

- Club empresas (20\%).

- ONG/Tercer Sector (20\%).

- Empleados $(20 \%)$.

Es el colectivo de aficionados, segmentados, los que ofrecen mayor relevancia, seguidos de patrocinadores y medios de comunicación. De estos grupos, los que cuentan con sección propia en la web son: peñas (95\%), accionistas (90\%), aficionados y fans $(85 \%)$ y abonados $(80 \%)$, patrocinadores $(60 \%)$ y demás grupos que aparecen de manera minoritaria, con menos del $25 \%$, como por ejemplo el colectivo de medios de comunicación. El contenido que parece en las secciones es meramente informativo y no responde a ningún planteamiento estratégico con objetivos o herramientas de comunicación específicos.

En cuanto a los grupos de interés de los clubes analizados en segunda división, encontramos que los principales grupos de interés que aparecen en los documentos analizados según la tabla de frecuencias son:

- Peñas $(100 \%)$.

- Aficionados (100\%).

- Abonados (100\%).

- Fans/seguidores (100\%).

- Medios de comunicación (94\%).

- Comunidad y sociedad en general (94\%).

- Accionistas $(64,7 \%)$.

- Patrocinadores $(58,8 \%)$.

- Club empresas $(29,4 \%)$.

- Administración local / gobierno (17,6\%).

- Sector educativo $(17,6 \%)$.

- Sector profesional $(11,8 \%)$.

- $\mathrm{ONG} /$ Tercer Sector $(5,9 \%)$.

- Empleados (5,9\%).

Se observa además cómo son los grupos relacionados con la afición y los medios de comunicación los recurrentes en todos los casos, seguidos de los accionistas y la comunidad en general. De estos grupos, los que cuentan con una sección propia en la web son las peñas (94\%), fans, aficionados y abonados ( $82 \%)$, medios de comunicación $(82 \%)$, accionistas $(41 \%)$, patrocinadores (35\%), club empresas (6\%), comunidad/sociedad $(6 \%)$. Al igual que en primera división, en segunda el contenido que parece en las secciones es meramente informativo y no responde a ningún planteamiento estratégico con objetivos o herramientas de comunicación específicos. 
En el análisis de las herramientas de comunicación disponibles, en el caso de la primera división (tabla 1) y segunda (tabla 2), se presenta a continuación:

Tabla 1. Herramientas Comunicación Primera División

\begin{tabular}{|l|l|}
\hline \multicolumn{1}{|c|}{ Herramienta } & $\begin{array}{l}\text { Frecuencia } \\
(\%)\end{array}$ \\
\hline Web corporativa & 100 \\
\hline Blog & 10 \\
\hline Foros (online) & 0 \\
\hline Buzón de sugerencias/reclamaciones/denuncias & 70 \\
\hline App & 50 \\
\hline Boletines online & 30 \\
\hline Microsite/portales específicos por públicos & 20 \\
\hline Foros, jornadas, encuentros & 80 \\
\hline Reuniones directas con colectivos, órganos reguladores, asociaciones,,.. & 90 \\
\hline Correo electrónico & 80 \\
\hline Revista Corporativa & 40 \\
\hline Canal TV propio & 40 \\
\hline Banco imágenes & 80 \\
\hline Twitter & 100 \\
\hline YouTube (canal) & 100 \\
\hline Flickr & 20 \\
\hline Facebook & 95 \\
\hline LinkedIn & 30 \\
\hline Instagram & 95 \\
\hline Snapchat & 15 \\
\hline RSS (suscripción de contenidos) & 50 \\
\hline $\begin{array}{l}\text { Otras Redes sociales (Google+, Weibo, WeChat, Viber, Pinterest, Line, } \\
\text { Dugout; Vimeo, etc.) }\end{array}$ & 70 \\
\hline
\end{tabular}

Tabla 2. Herramientas Comunicación Segunda División

\begin{tabular}{|l|l|}
\hline \multicolumn{1}{|c|}{ Herramienta } & $\begin{array}{l}\text { Frecuencia } \\
\text { \%) }\end{array}$ \\
\hline Web corporativa & 100 \\
\hline Blog & 5,9 \\
\hline Foros (online) & 0 \\
\hline Buzón de sugerencias/reclamaciones/denuncias & 58,8 \\
\hline App & 0 \\
\hline Boletines online & 5,9 \\
\hline Microsite/portales específicos por públicos & 11,8 \\
\hline
\end{tabular}




\begin{tabular}{|l|l|}
\hline \multicolumn{1}{|c|}{ Herramienta } & $\begin{array}{c}\text { Frecuencia } \\
(\%)\end{array}$ \\
\hline Foros, jornadas, encuentros & 0 \\
\hline Reuniones directas con colectivos, órganos reguladores, asociaciones, ... & 0 \\
\hline Correo electrónico & 100 \\
\hline Revista Corporativa & 41,2 \\
\hline Canal TV propio & 0 \\
\hline Banco imágenes & 76,5 \\
\hline Twitter & 100 \\
\hline YouTube (canal) & 100 \\
\hline Flickr & 17,6 \\
\hline Facebook & 94,1 \\
\hline LinkedIn & 5,9 \\
\hline Instagram & 100 \\
\hline Snapchat & 0 \\
\hline RSS (suscripción de contenidos) & 58,8 \\
\hline Otras Redes sociales (Google+, sportcam, Weibo , WeChat, MiaoHai) & 29,4 \\
\hline & \\
\hline
\end{tabular}

Otras herramientas empleadas por los clubes, que se identifican en la exploración de la presente investigación son:

- Sala de prensa.

- Entrevistas y reportajes bajo demanda (mención específica en la web).

- Radio online (directo y podcast).

- Encuestas.

- Social Wall.

- Descargas.

- WhatsApp para socios.

- Hemeroteca.

- Intranet para periodistas y socios.

- Programa para cada partido.

- Guía bienvenida aficionados.

- Sede Electrónica.

- $\quad$ Carteles anunciadores de partidos.

\subsection{RSE}

Sólo en tres casos emerge un microsite dedicado a la RSE (FC Barcelona y Deportivo Alavés de primera división, y la Unión Deportiva Almería SAD de segunda), pero sin información susceptible de ser analizada. 
Si bien, no se han encontrado secciones específicas donde los clubes afronten la RSE como un compromiso integrante de la gestión de la propia entidad, sí se identifican los temas o ámbitos de acción de la RSE con los que parecen estar más comprometidos (tablas 3 y 4):

\section{Tabla 3. Ámbitos RSE Primera División}

\begin{tabular}{|l|l|}
\hline \multicolumn{1}{|c|}{ Ámbitos } & Frecuencia (\%) \\
\hline Arte y Cultura & 45 \\
\hline Educación y formación & 80 \\
\hline Investigación, I+D+i, calidad, tecnología & 60 \\
\hline Familias & 75 \\
\hline Medio ambiente & 15 \\
\hline Pobreza y marginación /ayuda social & 75 \\
\hline Igualdad y conciliación (acciones puntuales día de la mujer) & 40 \\
\hline RR.HH./Empleados & 10 \\
\hline Ayuda en catástrofes & 0 \\
\hline Voluntariado (corporativo) & 25 \\
\hline Cooperación y solidaridad & 70 \\
\hline Salud (interna y externa) y seguridad & 15 \\
\hline $\begin{array}{l}\text { Integración y diversidad (inmigrantes, discapacitados, presos, } \\
\text { rehabilitados,...) }\end{array}$ & 55 \\
\hline Violencia en el deporte (violencia, racismo, xenofobia, ...) & 100 \\
\hline
\end{tabular}

Tabla 4. Ámbitos RSE Segunda División

\begin{tabular}{|l|l|}
\hline \multicolumn{1}{|c|}{ Ámbitos } & Frecuencia (\%) \\
\hline Arte y Cultura & 0 \\
\hline Educación y formación & 94,1 \\
\hline Investigación, I+D+i, calidad, tecnología & 17,6 \\
\hline Familias & 35,3 \\
\hline Medio ambiente & 5,9 \\
\hline Pobreza y marginación /ayuda social & 64,7 \\
\hline Igualdad y conciliación (acciones puntuales día de la mujer) & 29,4 \\
\hline RR.HH./Empleados & 0 \\
\hline Ayuda en catástrofes & 5,9 \\
\hline Voluntariado (corporativo) & 23,5 \\
\hline Cooperación y solidaridad & 94,1 \\
\hline
\end{tabular}




\begin{tabular}{|l|l|}
\hline \multicolumn{1}{|c|}{ Ámbitos } & \multicolumn{1}{|c|}{ Frecuencia (\%) } \\
\hline Salud (interna y externa) y seguridad & 64,7 \\
\hline $\begin{array}{l}\text { Integración y diversidad (inmigrantes, discapacitados, presos, } \\
\text { rehabilitados,...) }\end{array}$ & 76,5 \\
\hline Violencia en el deporte (violencia, racismo, xenofobia, ...) & 100 \\
\hline
\end{tabular}

\subsection{Fundaciones}

En el 100\% de los casos se deduce que las fundaciones son de titularidad privada. En primera división, el 60\% ofrece información económica de la entidad, el 40\% arroja información sobre los estatutos y su composición, así como información de sus empleados.

En ningún caso de segunda división se ofrece información económica de la fundación, ni se facilitan los estatutos ni datos de los empleados de la misma, si son diferentes a los del club con el que está vinculada. Sólo en el $20 \%$ se facilita la fecha de constitución y la composición del patronato de la misma.

Las áreas de interés para las fundaciones en primera división son: deporte $(100 \%)$, cultura $(70 \%)$, sociedad $(80 \%)$ e histórica $(65 \%)$. Otras temáticas encontradas son formación, juventud.

Las áreas de interés para las fundaciones de segunda división son deporte $(100 \%)$, cultura (70\%), sociedad (50\%) y formación (40\%). En otro orden de temáticas, encontramos alianzas internacionales, sostenibilidad e innovación social, solidaridad, salud y arte.

Los canales utilizados en primera división por las fundaciones para contactar, destacan el mail (45\%) y los perfiles de redes sociales propios (35\%), así como el teléfono (20\%). Entre las redes, destacan Facebook y Twitter (25\%), así como Instagram. Otros contenidos destacables son los formularios de respuesta, buzones de sugerencias, publicaciones online.

Entre los canales para contactar con la fundación en segunda división, destacan el email propio $(60 \%)$, microsite específico (70\%), Teléfono (40\%) y redes sociales propias (20\%). Entre las redes destacan Twitter (20\%), Facebook (20\%), YouTube (10\%) y Flickr (10\%). Otros contenidos destacables son los bancos de imágenes propios (20\%), las noticias $(40 \%)$ y los boletines $(20 \%)$.

\section{Discusión y conclusiones}

En las webs de los clubes de primera división emerge más profundidad de datos, puesto que los porcentajes, en la mayoría de los casos, son más altos en todos los parámetros analizados.

En el análisis de la información relativa a la Ley de la Transparencia, en amplia mayoría, aparece la información disponible y descargable, con mayor detalle de datos de composición del consejo de administración, detalles de remuneración (en muchos casos, explicitando que no existe), así como referencias al código ético.

Las webs de los clubes de fútbol español no están adaptadas según los protocolos de accesibilidad y por tanto, no son accesibles para personas con dificultades 
visuales. Se considera en este sentido conveniente remarcar la legislación existente en accesibilidad digital y que en todo caso se debería aplicar, concretamente:

- Ley 34/2002, de 11 de julio de 2002, de servicios de la sociedad de la información y comercio electrónico (LSSICE) ${ }^{7}$.

- Ley 51/2003, de 2 de diciembre de 2003, de igualdad de oportunidades, no discriminación y accesibilidad universal de las personas con discapacidad (LIONDAU) ${ }^{8}$.

Igualmente, es remarcable que en todos los casos se presenta la información cumpliendo con los apartados recogidos en la Ley de Transparencia, lo que trasmite que los clubes se limitan a cumplir con la ley y no tienen un compromiso real con la transparencia y la RSE, es decir, no van más allá.

Donde existe más similitud es en el análisis de los stakeholders y las herramientas de comunicación utilizadas en ambas categorías, ya que son segmentos de interés y fines muy coincidentes entre ambas divisiones. Las diferencias se acentúan, no obstante, en las redes sociales, donde los equipos de primera división poseen mayor número de ellas a disposición de sus aficionados, como respuesta a la demanda de información segmentada (Dugout), la madurez de sus estructuras corporativas (LinkedIn) y la internacionalización de sus mercados (Weibo), como algunos de los motivos que lo explican.

La similitud también es alta entre los objetivos dentro de la responsabilidad social empresarial de los clubes. Como tendencia, cabe destacar que los equipos de primera división están orientados a la formación, mientras que los de segunda división se esfuerzan en objetivos sociales y solidarios.

En las fundaciones de los clubes, encontramos mayor transparencia en la información, profundidad de estructura y variedad de herramientas de comunicación en las entidades de primera división, aunque es cierto que por otro lado, en segunda división se le presta atención especial a dichas entidades, hasta el punto de generar sites diferenciados en mayor medida. Independientemente de esto, resulta insuficiente la información facilitada en estas webs.

En el ámbito de la RSE, es relevante la ausencia de planes de RSE o memorias de RSE declaradas. Realmente, los clubes de fútbol no desarrollan políticas de RSE, sino más bien trabajan bajo el paraguas de la acción social o la filantropía y no son conscientes de los impactos que generan en la sociedad y su responsabilidad con la misma. Atreverse a enmarcarse dentro de un enfoque responsable, supone partir de la aceptación de que como organización generamos impactos de los que somos responsables, que debemos crear valor compartido y es nuestra obligación, y por tanto, supone un compromiso reducir los efectos negativos de nuestra actividad. Este planteamiento, está alineado con la estrategia renovada de la UE para la RSE publicada en 2011, donde se entiende por RSE a:

7 Disponible en: https://goo.gl/8pkFQ6

8 Disponible en: https://goo.gl/gGNcge 
La responsabilidad de las empresas por su impacto en la sociedad. (...) Para asumir plenamente su responsabilidad social, las empresas deben aplicar, en estrecha colaboración con las partes interesadas, un proceso destinado a integrar las preocupaciones sociales, medioambientales y éticas, el respeto de los derechos humanos y las preocupaciones de los consumidores en sus operaciones empresariales y su estrategia básica, a fin de:

- maximizar la creación de valor compartido;

- identificar, prevenir y atenuar sus posibles consecuencias adversas (Comisión Europea, 2011, p. 7).

Tras los datos expuestos, no se puede afirmar que los clubes de fútbol españoles apuestan por la RSE como un compromiso con la sociedad y sus impactos, asumiendo su nuevo rol como ciudadano. Todo lo contrario. Mientras los ingresos siguen aumentando (3327 millones, temporada 2016/2017 ver figura 1), la deuda con la Hacienda española disminuye, pero no en la misma proporción que aumentan los ingresos, ya que aún adeudan a las arcas públicas 184 millones de euros (Mazo et al., 2017). No podemos obviar que los clubes de fútbol españoles tienen un impacto en nuestra sociedad y a tenor de la definición expuesta, el concepto de RSE no es aplicable.

Figura 1. Ingresos de La Liga (Expansión, 2017)

\section{EVOLUCIÓN DE LOS INGRESOS DE LA LIGA POR CÁPITULOS}

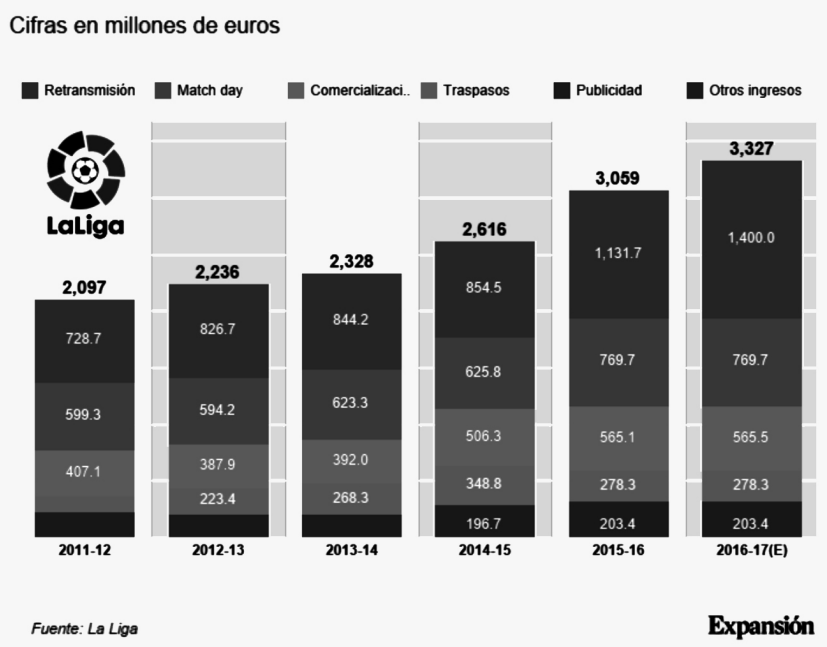

Fuente: Expansión ${ }^{9}$

La Liga, como patronal de las entidades o clubes que componen la competición profesional, abandera un compromiso aprobado en su asamblea de forma unánime y comprometida. No obstante, los datos que refleja nuestro estudio indican que la política de acción de cada uno de los clubes está aún lejos de cumplir con su cuota particular

9 Disponible en: https://goo.gl/mL9hgX 
de responsabilidad defendido por el ente, que proyecta unos compromisos globales más elevados que la suma particular de los miembros que la componen.

A la luz de la hipótesis de partida, que planteaba que los clubes apuestan por acciones de RSE como una vía para legitimar su rol en la sociedad como ciudadano corporativo, podemos afirmar que ésta no se ve confirmada. Podemos concluir con una reflexión no del todo catastrofista, al plantear que los clubes intentan legitimar su rol en la sociedad con políticas sociales, pero no sería pertinente delimitarlas como RSE, sino como acción social.

\section{Referencias}

Asociación Española de Fundaciones (s.f.). La fundación: concepto, constitución y régimen sustantivo y tributario. (https://bit.ly/2beLnUS).

Ángel-Vega, J. L. (2009). Responsabilidad Social y los principios del desarrollo sostenible como fundamentos teóricos de la información social de la empresa. Madrid: ESIC.

Cano-Tenorio, R. \& Paniagua-Rojano, F.J. (2017). El uso de Twitter por parte de los futbolistas profesionales. Contenidos y relaciones con los públicos. [The use of Twitter by the professional football players. Contents and relations with the publics relations]. Revista Internacional de Relaciones Públicas, 7(13), 101-122. (https://goo.gl/AXqUG2)

Cano-Tenorio, R. (2017). Técnicas de marketing de los clubes de futbol de élite en las redes sociales. [Marketing techniques of the elite football clubs in the social media]. Retos, 7(13), 43-58. http://dx.doi.org/10.17163/ret.n13.2017.03.

Capriotti, P. (2006). Concepción e importancia actual de la Ciudadanía Corporativa. Razón y Palabra, 53. (https://bit.ly/2IyVYsL) (13/03/2018).

Castillo, A., Fernández, M. \& Castillero, E. (2016). Fútbol y redes sociales. Análisis de la gestión de Relaciones Públicas 2.0 por los clubes de fútbol. Estudios sobre el Mensaje Periodístico, 22(1), 239-254. http://dx.doi.org/10.5209/rev_ESMP.2016.v22.n1.52592.

Castillo, A. (2009). Relaciones Públicas. Teoría e Historia. Barcelona: UOC.

Carroll, A. B. (1999). Corporate Social Responsibility. Evolution of a definition. Business and Society, 38(3), 268-295. https://doi.org/10.1177/000765039903800303.

Comisión Europea (2011). Estrategia renovada de la UE para 2011-2014 sobre la responsabilidad social de las empresas. (https://bit.ly/2KdBrQ0).

Cortina, A. (1994). Ética de la empresa. Madrid: Trotta.

Freeman, R. E. (1984). Strategic Management: A Stakeholder Approach. Boston: Pitman Publishing.

Freeman, R. E., Harrison, J. S., Wicks, A.C., Parmar, B. y Colle, S. (2010). Stakeholder Theory. The State of the Art. Nueva York: Cambridge University Press.

García-Marzá, D. (2007). Responsabilidad social de la empresa: una aproximación desde la ética empresarial. Veritas: Revista de Filosofía y Teología, 2(17), 183-204. (https://bit.ly/2IAhYn1) $(13 / 03 / 2018)$.

Gobierno de España (2013). Ley 19/2013, de 9 de diciembre, de Transparencia, Acceso a la Información Pública y Buen Gobierno. Madrid: Ministerio de la presidencia. (https://bit.ly/2KhvzFC).

Gobierno de España (1999). Real Decreto 1251/1999, de 16 de julio, sobre sociedades anónimas deportivas. Madrid: Ministerio de la presidencia. (https://bit.ly/2Kur9Hi).

Gobierno de España (1990). Ley 10/1990, de 15 de octubre, del Deporte. Madrid: Ministerio de la presidencia. (https://bit.ly/2yPSMtf).

Grunig, J. E. \& Hunt, T. (2003). Dirección de Relaciones Públicas. Barcelona: Gestión 2000.

Instituto Ethos de Empresas y Responsabilidad Social (2017). Indicadores Ethos. Glossário. (https:// bit.ly/2yMHZQg).

La Liga (2015). Guía Práctica para Aficionados. (https://bit.ly/2KelCbT). 
La Liga (2015). Guía de Buenas Prácticas. Normativa y principios para las federaciones, ligas y deportistas españoles. (https://bit.ly/2tLvfUB).

La Liga (2017). Mapa de la responsabilidad social del fútbol profesional español. (https://bit.ly/2MvT8Hk).

Lindblom, C. K. (1994). The implications of organisational legitimacy for corporate social performance and disclosure. Presentado en Critical Perspectives on Accounting Conference, Nueva York.

López-Martínez, R. \& Fernández-Fernández, J.L. (2015). Responsabilidad Social Corporativa y Buen Gobierno en los clubes de fútbol españoles. [Corporate Social Responsibility and Good Governance in Spanish football clubs]. Universia Business Review, (46), 38-53. (https://bit.ly/2N8I5EW) $(13 / 03 / 2018)$.

Mazo, E.; Junco. L.; Cruzado, V. \& Galera, C. (2017). Así están las finanzas del fútbol español: ingresos récord y deuda en mínimos. En Expansión. Deporte y Negocio. (https://bit.ly/2lCSpst) $(13 / 03 / 2018)$.

Navarro, F. (2008). Responsabilidad Social Corporativa: Teoría y práctica. Madrid: ESIC.

O'Donovan, G. (2002). Environmental disclosures in the annual report: Extending the applicability and predictive power of legitimacy theory. Accounting, Auditing $\mathcal{E}$ Accountability Journal, 15(3), 344-371. https://10.1108/09513570210435870.

Olabe, F. (2012). La RSC como factor de reputación en los clubes de fútbol profesionales. [CSR as a reputation factor for profesional football clubs]. Revista Internacional de Investigación en Comunicación aDResearch ESIC, 6(6), 144-157. https://doi.org/10.7263/ADR.RSC.006.08.

Raufflet, E., Lozano, J.F., Barrera, E. \& García, C. (2012). Responsabilidad Social Empresarial. México: Pearson.

Silva-Santos, B., Molina-Moreno, V., Gutiérrez, L., \& Carvalho, M. J. (2014). Revisâo sistematica sobre a Responsabilidade Social Corporativa e o desporto. Revista Intercontinental de Gestâo Desportiva, (4) (Supl. I), 126-146. (https://bit.ly/2IADA2z) (13/03/2018).

Seitel, F. (2002). Teoría y práctica de las Relaciones Públicas. Madrid: Pretencie Hall.

Shocker, A. D. \& Sethi, S. P. (1973). An approach to developing societal preferences in developing corporate action strategies. California Management Review, 15(4), 97-105.https://doi. org/10.2307/41164466.

UEFA (2017). Responsabilidad Social. (https://bit.ly/2lE2oxB).

Wang, J. \& Chaudhri, V. (2009). Corporate social responsibility engagement and communication by Chinese companies. Public Relations Review, (35), 247-250.https://10.1016/j.pubrev.2009.04.005. 